\title{
Highly Conductive Metallic State and Strong Spin-Orbit Interaction in Annealed Germanane
}

\author{
Qihong Chen, ${ }^{\dagger}{ }^{\circ}$ Lei Liang, ${ }^{\dagger}$ Georgia Potsi, ${ }^{\ddagger}$ Puhua Wan, ${ }^{\dagger}$ Jianming Lu, ${ }^{\dagger, \S}$ Theodosis Giousis, ${ }^{\ddagger}, \|$ \\ Eleni Thomou, ${ }^{\ddagger, \|}$ Dimitrios Gournis," Petra Rudolf, ${ }^{\ddagger \odot}$ and Jianting Ye, ${ }^{* \dagger}$
}

\begin{abstract}
${ }^{\dagger}$ Device Physics of Complex Materials, Zernike Institute for Advanced Materials, University of Groningen, Nijenborgh 4, 9747 AG Groningen, The Netherlands

${ }^{\ddagger}$ Surfaces and Thin Films, Zernike Institute for Advanced Materials, University of Groningen, Nijenborgh 4, 9747 AG Groningen, The Netherlands

${ }^{\S}$ State Key Laboratory for Mesoscopic Physics, Department of Physics, Peking University, No. 5 Yiheyuan Road Haidian District, 100871 Beijing, P. R. China

"Department of Materials Science \& Engineering, University of Ioannina, 45110 Ioannina, Greece
\end{abstract}

Supporting Information

ABSTRACT: Similar to carbon, germanium exists in various structures such as three-dimensional crystalline germanium and germanene, a two-dimensional germanium atomic layer. Regarding the electronic properties, they are either semiconductors or Dirac semimetals. Here, we report a highly conductive metallic state in thermally annealed germanane (hydrogen-terminated germanene, $\mathrm{GeH}$ ), which shows a resistivity of $\sim 10^{-7} \Omega \cdot \mathrm{m}$ that is orders of magnitude lower than any other allotrope of germanium. By comparing the resistivity, Raman spectra, and thickness change measured by AFM, we suggest the highly conductive metallic state is associated with the dehydrogenation during heating, which likely transforms germanane thin flakes to multilayer germanene. In addition, weak antilocalization is observed, serving as solid evidence for strong spin-orbit interaction (SOI) in germanane/germanene. Our study opens a possible new route to investigate the electrical transport properties of germanane/germanene, and the large SOI might provide the essential ingredients to access their topological states predicted theoretically.

KEYWORDS: Germanane, multilayer germanene, dehydrogenation, metallic state, weak antilocalization, strong spin-orbit interaction

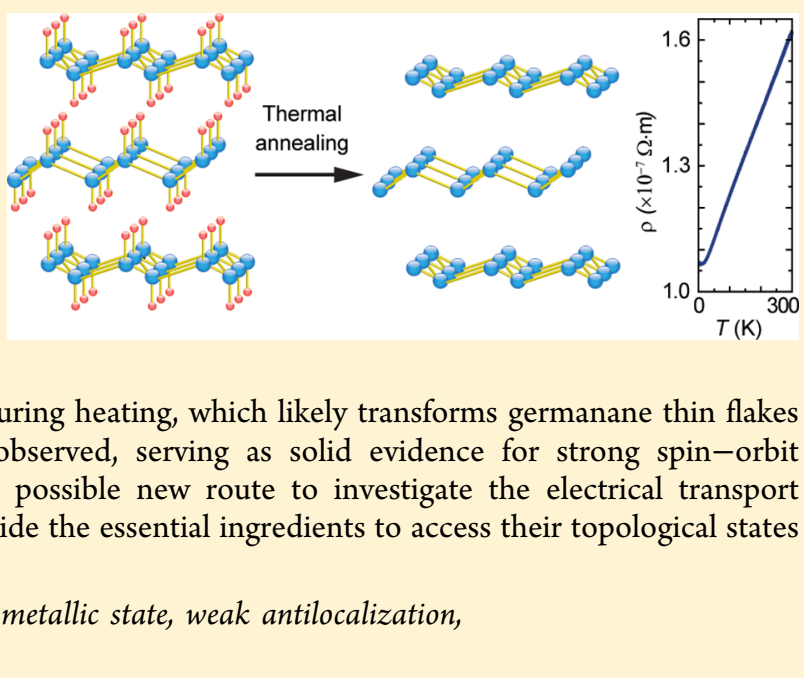

A tomically thin two-dimensional (2D) materials such as graphene, ${ }^{1,2}$ phosphorene, ${ }^{3}$ transition metal dichalcogenides, ${ }^{4-11}$ etc., are regarded as promising candidates to replace conventional silicon in the next generation nanoelectronics. $^{12-16}$ Among them, 2D monolayers composed of group IV elements such as silicene ${ }^{17-23}$ and germanene $e^{24-28}$ are of particular interest because they are fully compatible with the well-established protocols for device fabrication and integration in the present silicon- and germanium-based semiconductor technologies. Similar to graphene, monolayer silicon and germanium, i.e., silicene and germanene, are both Dirac semimetals, whose band structure exhibits crossings at the $K / K^{\prime}$ points of the Brillouin zone with zero energy gap. This hallmark Dirac band structure is the origin of massless carriers, making graphene the most studied material in recent years. However, the gapless electronic structure is not favorable for making a transistor, therefore limiting the application of these Dirac materials in real devices. Compared with very stable graphene, silicene and germanene are not stable in air, which is another drawback for device applications.
Recently, hydrogen termination on graphene has been proved to be an effective way to open a band gap. ${ }^{29,30}$ Similarly, germanane (i.e., hydrogen-terminated germanene, $\mathrm{GeH}$ ) was synthesized by adding covalently bonded hydrogen on both sides of germanene. ${ }^{31}$ Since the $\mathrm{p}_{z}$ orbitals of $\mathrm{Ge}$ bond covalently to the $\mathrm{H}$ atoms, the contribution of the $\mathrm{p}_{z}$ orbitals to electron transport is significantly suppressed and a direct band gap opens at the $\Gamma$ points of the Brillouin zone. ${ }^{32-38}$ As a result, a conduction band derived from the sorbitals and a valence band derived from the $\mathrm{p}_{x}$ and $\mathrm{p}_{y}$ orbitals near the $\Gamma$ point determine the transport properties. A high electron mobility is expected owing to the low effective mass. ${ }^{31}$ The large band gap, high mobility, and low dimensionality make germanane very attractive for short-channel field effect transistors (FETs). Furthermore, the $\sigma$ bond (composed of $\mathrm{p}_{x}$ and $\mathrm{p}_{y}$ orbitals) that dominates the electron transport has a

Received: October 18, 2018

Revised: January 5, 2019

Published: January 24, 2019 

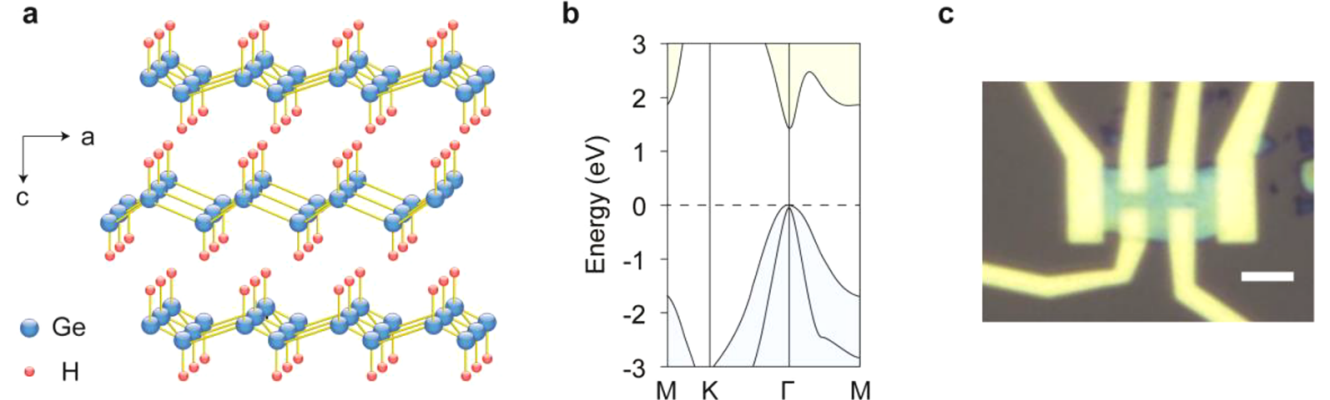

Figure 1. Germanane characteristics and device. (a) Schematic illustration of the crystal structure of germanane. Germanium atoms form a buckled honeycomb lattice in the $a b$-plane, and each germanium atom is bonded with one hydrogen atom in the $c$-direction. (b) Simplified electronic band structure of monolayer germanane. Reproduced from theoretical calculations of ref 35. (c) Optical image of a typical device on $\mathrm{SiO}_{2}(300 \mathrm{~nm}) / \mathrm{Si}$ $\left(\mathrm{n}^{++}\right)$substrate. Scale bar: $3 \mu \mathrm{m}$.
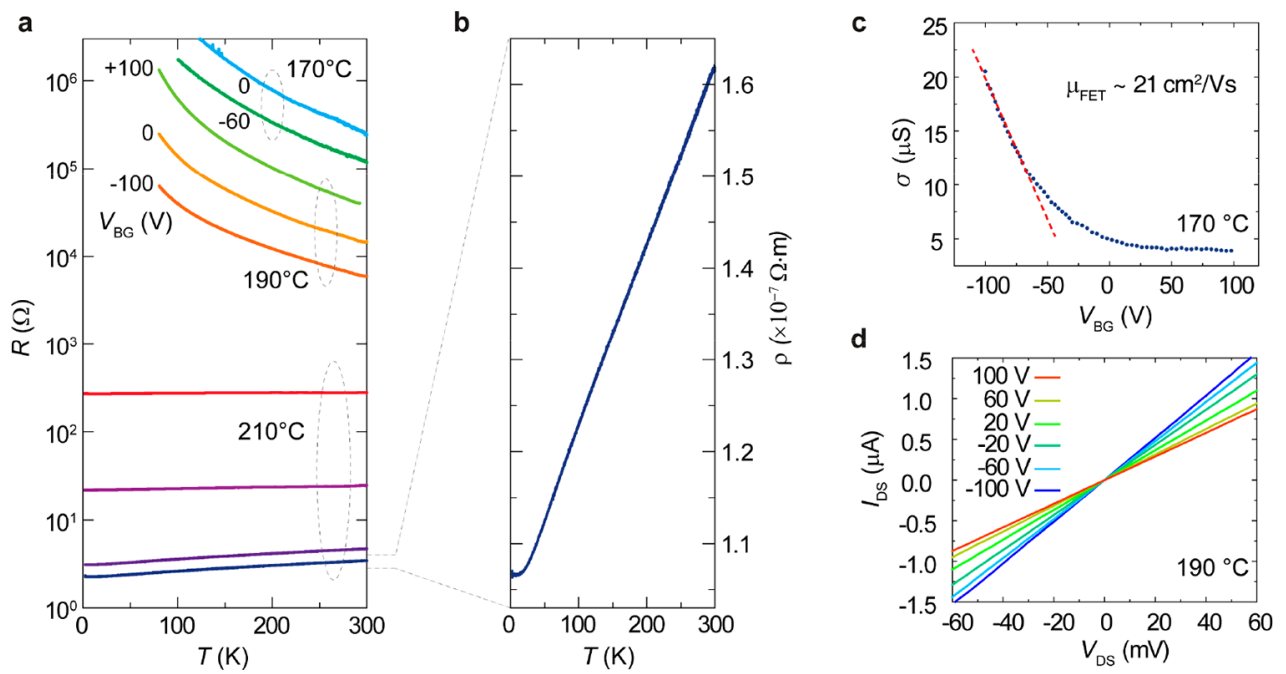

Figure 2. Insulator-to-metal transition via thermal annealing. (a) Temperature dependence of the germanane channel resistance after annealing at different temperatures, in log scale. The top two dashed circles represent results from two devices annealed at 170 and $190{ }^{\circ} \mathrm{C}$, respectively. Different curves in each circle are measured with different back-gate voltages $V_{\mathrm{BG}}(\mathrm{V})$. The curves marked by the bottom circle are measured from three different devices after annealing at $210^{\circ} \mathrm{C}$. (b) Temperature dependence of the resistivity, corresponding to the lowest curve in (a), in linear scale. (c) Transfer curve of the device indicated by the top dashed circle in panel (a) measured at $T=300 \mathrm{~K}$, showing a p-type transistor operation. The red dashed line indicates the linear fitting in the switch-on region, corresponding to a mobility of $21 \mathrm{~cm}^{2} \mathrm{~V}^{-1} \mathrm{~s}^{-1}$. (d) $I-V$ characteristics of the device indicated by the middle dashed circle in panel (a) for different back gate voltages, measured at $T=300 \mathrm{~K}$.

stronger spin-orbit interaction (SOI) than the original $\pi$ bond (composed of $\mathrm{p}_{z}$ orbitals); therefore, a nontrivial spin-orbit gap of $\sim 0.2 \mathrm{eV}$ opens in the valence band. ${ }^{31,39}$ Combined with the large direct band gap, the traditional spin-selective optical process is also expected to be present in germanane, making it an attractive candidate for optoelectronic applications.

Despite the interesting properties discussed previously, very limited work has been reported regarding its electrical transport properties. ${ }^{40-42}$ In this study, we fabricated field effect transistors based on germanane thin flakes and focused on the transport properties at low temperatures and under magnetic fields. The as-grown germanane shows very low conductivity, which agrees with the large band gap. ${ }^{31,33,34}$ After the germanane was annealed in an inert gas atmosphere, we observe a drastic increase of the conductivity with a clear transition from insulator to metal. A p-type field effect transistor is realized on the annealed germanane. Combining the Raman spectra, AFM, and electrical transport, we suggest that the metallic behavior is likely attributed to a dehydrogenation process, which maintains the layered structure. ${ }^{23}$ In addition, a clear weak antilocalization (WAL) feature is observed at low temperatures, indicating strong spin-orbit interaction in annealed germanane. These explorations open new opportunities for quantum and spin transport studies of germanane for both basic research and device applications.

Bulk germanane crystals were synthesized following the recently developed topotactic deintercalation method, as detailed in ref 42 . In this method, $\mathrm{CaGe}_{2}$ was first prepared by the reaction of high purity germanium $(\mathrm{Ge})$ and calcium (Ca) in a vacuum at $950-1050{ }^{\circ} \mathrm{C}$. Germanane was then synthesized following an exchange process between the obtained $\mathrm{CaGe}_{2}$ and $\mathrm{HCl}$, where $\mathrm{Ca}$ atoms in $\mathrm{CaGe}_{2}$ are substituted by $\mathrm{H}$ atoms. This chemical process can be described by the equation $\mathrm{CaGe}_{2}+2 \mathrm{HCl} \rightarrow 2 \mathrm{GeH}+\mathrm{CaCl}_{2}$.

As shown in Figure 1a, a monolayer $\mathrm{GeH}$ has a honeycomb lattice, where each $\mathrm{Ge}$ is covalently bonded with another three $\mathrm{Ge}$ atoms in the $a b$-plane and one $\mathrm{H}$ atom in the $c$-direction for $\mathrm{sp}^{3}$ hybridization. The single-crystal X-ray diffraction (XRD) shows that the unit cell is slightly distorted from the expected hexagonal lattice, becoming monoclinic with lattice parameters $a=6.924 \AA, b=3.998 \AA, c=10.939 \AA$, and $\beta=102.181^{\circ} .42$ The $a b$-plane covalent bonds between Ge atoms are buckled; 
a

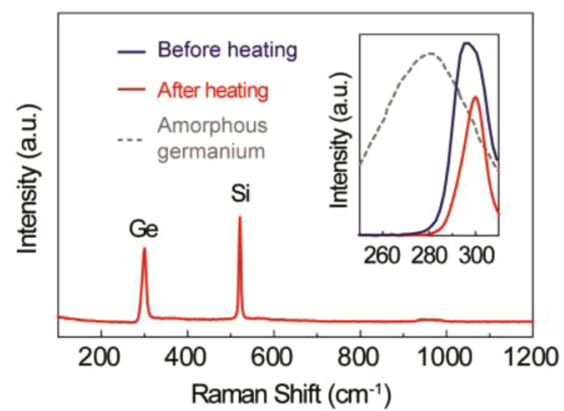

b

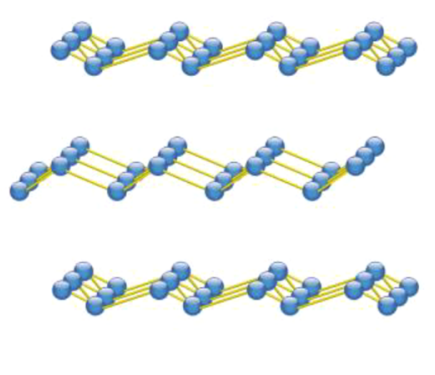

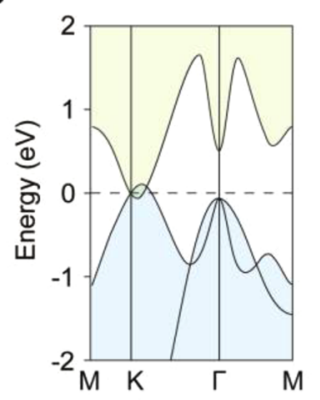

d

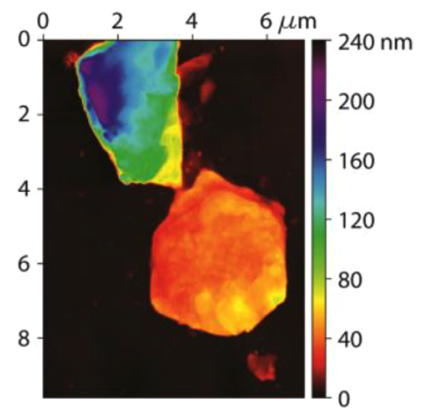

e

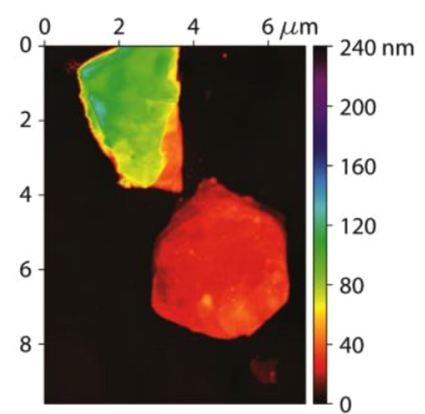

$\mathbf{f}$

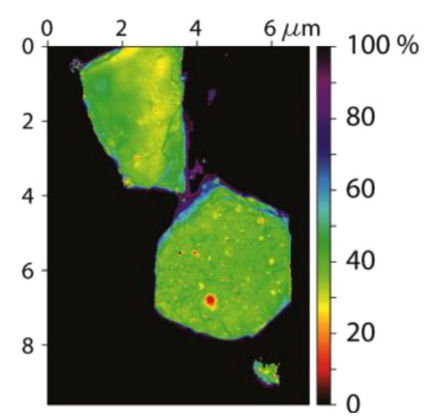

Figure 3. From germanane to germanene. (a) Raman spectra of annealed germanane. Inset: blow up of the region for the $\mathrm{Ge}-\mathrm{Ge}$ vibrational mode of as-prepared germanane (dark brown), annealed germanane (red), and hydrogenated amorphous germanium (dashed gray)..$^{44}$ (b) Schematic illustration of the possible crystal structure after annealing, multilayer germanene. (c) Simplified electronic band structure of bilayer germanene, with valence and conduction band crossing around $K$ point. Reproduced from theoretical calculations of ref 28. (d) - (f) AFM images of germanane before annealing, after annealing at $250{ }^{\circ} \mathrm{C}$, and the reduction of height in percentage, respectively. The spot in the top middle part with height reduction of $\sim 100 \%$ is the tape residue, which is completely removed after the annealing.

i.e., the two triangular sublattices are displaced relative to each other in the $c$-direction. The stacking of buckled $a b$-planes forms a layered structure with an interlayer distance of $5.5 \AA$. Depending on the stacking sequence, the $\mathrm{GeH}$ has different polytypes of $1 \mathrm{~T}, 2 \mathrm{H}$, and $6 \mathrm{R}^{43}$ The additional hydrogen bonding in the $c$-direction significantly influences the electronic structure. Figure $1 \mathrm{~b}$ shows a simplified band structure of $\mathrm{GeH},{ }^{31,35}$ where a direct band gap of $\sim 1.6 \mathrm{eV}$ presents at the $\Gamma$ point of the Brillouin zone. Chemically, while germanene is highly reactive with oxygen and therefore is normally only stable in ultrahigh vacuum or inert gas atmosphere, $\mathrm{GeH}$ becomes stable at ambient conditions, which is important for the following study of electrical transport. Similar to other layered materials, germanane thin flakes were prepared by mechanical exfoliation of bulk crystals on highly doped $\mathrm{Si}$ substrate coated with $300 \mathrm{~nm} \mathrm{SiO}$. Optical microscope and AFM were used to select uniform and thin flakes. We chose typical $\mathrm{GeH}$ flakes with thicknesses of 20-50 $\mathrm{nm}$ for device fabrication and subsequent electrical measurements. The electrodes, composed of $\mathrm{Ti} / \mathrm{Au}(5 / 65 \mathrm{~nm})$, were patterned in a Hall bar geometry via the electron-beam lithography. Figure 1c shows a typical optical image of a $\mathrm{GeH}$ device. The low temperature measurements were performed using either a Janis liquid nitrogen system or cryostat with a base temperature of $2 \mathrm{~K}$ and a $12 \mathrm{~T}$ magnet. Resistance was measured with a constant ac current excitation using standard lock-in amplifiers (Stanford Research SR830), and the gate voltages were set by a dc source meter (Keithley 2450).

The as-prepared device is very insulating, with room temperature sheet resistance $R_{\mathrm{s}}$ well above $10 \mathrm{M} \Omega$ (input impedance of the lock-in amplifier). The highly resistive state is consistent with its semiconducting nature and finite band gap (Figure 1b). After the initial characterization, the device was annealed in a tube furnace with a continuous follow of $\mathrm{Ar}$ gas $(100 \mathrm{sccm})$ at ambient pressure. The annealing temperature was linearly ramped up to the target within $1-2 \mathrm{~h}$ and kept for $12 \mathrm{~h}$ before naturally cooling down. Figure 2a summarizes the temperature dependence of resistance after annealing. The resistance reduces to below $1 \mathrm{M} \Omega$ right after annealing at $170{ }^{\circ} \mathrm{C}$. The temperature dependence of the resistance $(R T)$ reveals an insulating behavior, where the resistance increases with decreasing temperature. Remarkably, the sample exhibits a clear field effect after annealing. As can be seen in Figure $2 c$, the transfer curve shows a p-type transistor operation where the conductivity $\sigma$ increases by $\sim 5$ times at a back gate voltage of $V_{\mathrm{BG}}=-100 \mathrm{~V}$. The FET mobility can be calculated from the geometric capacitance: $\mu_{\mathrm{FET}}=\frac{1}{C_{\mathrm{g}}} \frac{\mathrm{d} \sigma}{\mathrm{d} V_{\mathrm{BG}}}$, where $\sigma$ is the conductivity and $C_{\mathrm{g}}=11 \mathrm{nF} / \mathrm{cm}^{2}$ is the gate capacitance per unit area for $300 \mathrm{~nm} \mathrm{SiO}$. By the linear fitting of the gate dependence of conductivity, the extracted FET mobility reaches $21 \mathrm{~cm}^{2} \mathrm{~V}^{-1} \mathrm{~s}^{-1}$ at room temperature.

When the annealing temperature is raised to $190{ }^{\circ} \mathrm{C}$, a significant increase in the conductivity is observed, as can be seen from the three curves labeled by the middle dashed circle in Figure 2a. Figure $2 \mathrm{~d}$ shows the corresponding $I-V$ characteristics of the same device measured at different back gate voltages. A consistent p-type transistor behavior is observed, as the conductivity increases at negative $V_{\mathrm{BG}}$. In addition, all $I-V$ characteristics show perfectly linear dependence, suggesting ohmic contacts between electrodes and germanane flakes. Although the overall conductivity increases 
significantly compared to the sample annealed at $170{ }^{\circ} \mathrm{C}$, the $R T$ dependence still shows insulating behavior.

Only after the annealing temperature is further raised to 210 ${ }^{\circ} \mathrm{C}$ does the resistance decrease drastically. In contrast to the previous insulating states, a typical metallic behavior is observed, where $R_{\mathrm{s}}$ decreases with the decrease of temperature (Figure 2b). This enhanced metallicity is also accompanied by the loss of tunability by the electric field, i.e. the solid state back gate cannot tune the source-drain current $I_{\mathrm{DS}}$ or the channel resistance. Employing the stronger ionic gating technique, ${ }^{5,7,10}$ the $I_{\mathrm{DS}}$ can be slightly tuned but the effect is also very weak (see Figure S4). The annealed germanane behaves like a piece of metal.

Germanane itself is unlikely to be turned into a metallic state because of the large band gap. The highly conductive state induced by thermal treatment immediately brings to mind the metallic state predicted for germanene, which has never been measured by electrical transport. To get a better understanding of this behavior, we measured the Raman spectra before and after thermal annealing. As shown in Figure 3a, the main peak at $\sim 300 \mathrm{~cm}^{-1}$ can be assigned to the $E_{2}$ mode of $\mathrm{Ge}-\mathrm{Ge}$ vibration in the $a b$-plane. ${ }^{31}$ This feature is very close to the $\mathrm{Ge}-\mathrm{Ge}$ stretching mode of 3D crystalline germanium at 297 $\mathrm{cm}^{-1}$, suggesting that attaching light $\mathrm{H}$ atoms has little influence on the vibration of $\mathrm{Ge}-\mathrm{Ge}$. After heating, the peak line width reduces from a fwhm (full width at half-maximum) of 18 to $10 \mathrm{~cm}^{-1}$. As a reference, the gray dashed line in the inset of Figure $3 \mathrm{a}$ shows the $\mathrm{Ge}-\mathrm{Ge}$ vibrational mode of hydrogenated amorphous germanium. ${ }^{44-47}$ In sharp contrast, the $\mathrm{Ge}-\mathrm{Ge}$ mode centered around $270 \mathrm{~cm}^{-1}$ is very broad with a fwhm of $\sim 50 \mathrm{~cm}^{-1}$. This comparison rules out the formation of conventional amorphous germanium after annealing. On the contrary, the even narrower peak suggests higher crystallinity compared with the pristine $\mathrm{GeH}$ single crystal. The identical vibrational mode also indicates that the nature of bonding in the $a b$-plane is preserved. Consequently, we expect no change in the layered structure after annealing.

From the transport measurement, we are able to calculate the resistivity of germanane after annealing. The most conductive sample shows a resistance of $\sim 3 \Omega$ at room temperature, with channel dimensions of $1.6 \mu \mathrm{m}$ (length) $\times$ $2.5 \mu \mathrm{m}$ (width) $\times 30 \mathrm{~nm}$ (thickness). The calculated resistivity is $1.6 \times 10^{-7} \Omega \cdot \mathrm{m}$. Table 1 lists the room temperature resistivity for different materials related to our analysis.

For bulk crystalline and amorphous germanium, the resistivity at room temperature is on the order of $0.1-1 \Omega$. $\mathrm{m}$, and the temperature dependences are dominated by the semiconducting gap and variable range hopping, where resistivity increases rapidly with the decrease of temper-

Table 1. Room Temperature Resistivity for Crystalline Germanium, ${ }^{48}$ Amorphous Germanium, ${ }^{49}$ Heavily Doped Germanium, ${ }^{50}$ Graphite, ${ }^{51}$ Annealed Germanane, and Graphene $^{52}$

\begin{tabular}{ll}
\multicolumn{1}{c}{ material } & resistively $(\Omega \cdot \mathrm{m})$ \\
germanium (crystalline) & 0.1 \\
germanium (amorphous) & 1 \\
germanium (heavily doped) & $2 \times 10^{-6}$ \\
graphite & $6 \times 10^{-6}$ \\
annealed GeH & $1.6 \times 10^{-7}$ \\
graphene & $1 \times 10^{-8}$
\end{tabular}

ature. $^{49,53-55}$ From the observed metallic behavior, we can safely rule out the possibility that the annealed germanane turned into conventional crystalline or amorphous germanium. A quasi-metallic behavior can be obtained in heavily doped germanium, ${ }^{50,56}$ in which the resistivity of $\sim 2 \times 10^{-6} \Omega \cdot \mathrm{m}$ is still 1 order of magnitude higher than our annealed germanane. Therefore, reaching the present state of low resistivity would require even higher doping, which should leave spectral signatures that are easily detectable with standard elemental analysis on degenerate-doped semiconductors. However, the combined XPS and EDX analysis (see Figure S1 of the Supporting Information) shows no trace of impurity doping. Considering the sharper Raman peak after annealing, and high quality initial single crystal of $\mathrm{GeH}$, the possible production of heavily doped crystalline germanium can also be excluded. In Table 1, we can see that the resistivity of annealed germanane is even lower than graphite, and only higher than monolayer graphene. Therefore, the observed significant increase in conductivity is very likely associated with the dehydrogenation of $\mathrm{GeH}^{31}$ The heat treatment can break the $\mathrm{Ge}-\mathrm{H}$ bond, and hydrogen atoms are released from the lattice, possibly leaving behind a multilayer germanene structure, as schematically shown in Figure 3b.

As aforementioned, the two triangular sublattices of germanene are slightly displaced with respect to each other in the $c$-direction. The buckling parameter $\Delta$, i.e., the separation between the two sublattices in the $c$-direction, is determined by the competition between the electronic and elastic energies. For free-standing monolayer germanene, the $\Delta$ calculated by density functional theory (DFT) is $\sim 0.65 \AA .^{57}$ With this buckling, gemanene is a $2 \mathrm{D}$ Dirac semimetal whose band structure exhibits a crossing at the $K / K^{\prime}$ points with a zero density of states. Although energetically unfavorable, the Fermi levels of weakly buckled $(\Delta<0.5 \AA)$ and highly buckled monolayer germanene $(\Delta>0.7-0.8 \AA)$ cross bands below the $K / K^{\prime}$ points with finite density of states. In annealed multilayer germanene, it is possible that the buckling of germanene deviates from the ideal free-standing value due to the interlayer interaction as well as the interaction with the underlying substrate, hence inducing metallic state with higher density of states. Consistently, calculations show that a free-standing bilayer germanene has a nonzero density of states at the Fermi level (Figure 3c). ${ }^{28}$ This simple trend has been observed when graphene is stacked to form graphite. Similarly, a high density of states at the Fermi level is expected for multilayer germanene; hence, high electrical conductivity is expected.

It should be mentioned that dehydrogenation is also observed in a previous study ${ }^{31}$ and the temperature lies between 200 and $250{ }^{\circ} \mathrm{C}$, which is higher than our observation of $170-210{ }^{\circ} \mathrm{C}$. In our experiments, we observed a strong thickness dependence of the annealing temperature necessary to induce the insulator-metal transition; i.e., following the identical procedure, thicker flakes require a higher temperature to reach the metallic state. Therefore, it is consistent that the previous dehydrogenation of bulk crystals required higher temperature. In the present study, the heating temperatures shown in Figure 2a refer to flakes that are around $30-50 \mathrm{~nm}$ thick.

This dehydrogenation scenario is further supported by our AFM study, as shown in Figures $3 \mathrm{~d}-\mathrm{f}$. In multilayer germanane, the layers are bonded by the weak van der Waals forces, with an interlayer distance of $5.5 \AA$. The interlayer interaction is of comparable strength to the intralayer 


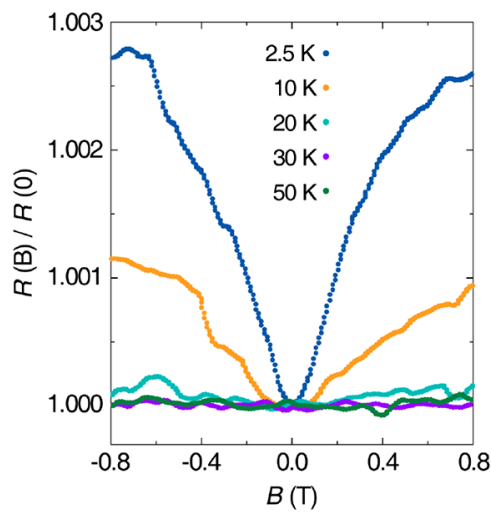

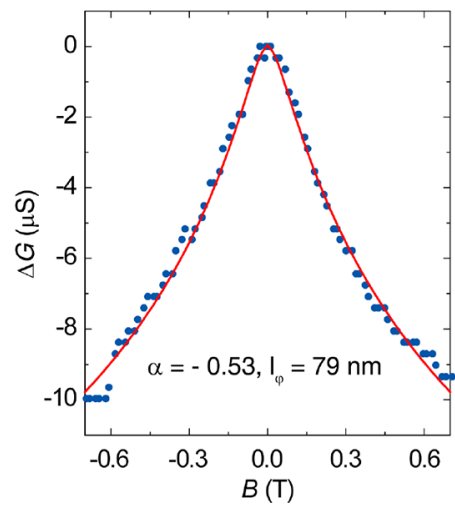

Figure 4. Evidence for strong spin-orbit interaction. (a) Normalized magnetoresistance of an annealed germanane device at different temperatures, showing clear weak antilocalization (WAL) characteristics at low field region. (b) Measured magnetoconductance (dots), which is defined as $\Delta G=G(B)-G(0)$ at $T=2.5 \mathrm{~K}$. The red solid line is the best fitting to $\Delta G$ using the WAL equation $(1)$, with $\alpha=-0.53$ and phase coherence length $l_{\Phi}=79 \mathrm{~nm}$.

interactions, which is rather strong. ${ }^{28}$ Consequently, the interlayer distance of germanene is much smaller than that of germanane. This has been confirmed previously by STM studies that show large flat terraces with steps of about $3.2 \AA$ in few-layer germanene flakes grown on a gold template ${ }^{26}$ and on an $\mathrm{MoS}_{2}$ subtrate. ${ }^{27}$ Comparing the AFM height profile before (Figure 3d) and after (Figure 3e) annealing at $250{ }^{\circ} \mathrm{C}$, we observed a significant decrease in thickness. Here, we chose thicker flakes and higher annealing temperature $\left(250{ }^{\circ} \mathrm{C}\right.$ instead of $210{ }^{\circ} \mathrm{C}$ used in Figure 2a) for complete removal of hydrogen. Figure $3 \mathrm{f}$ shows a $2 \mathrm{D}$ map of the reduction of thickness in percentage. In spite of very different initial thicknesses in different locations, a uniform reduction of $\sim 40 \%$ in thickness was observed over the entire flake. Considering the change of interlayer distance, $5.5-3.2 \AA / 5.5 \AA \approx 42 \%$, the observed uniform reduction of $\sim 40 \%$ is highly consistent with the difference of interlayer distance between germanane and germanene. Therefore, this direct measurement strongly supports the dehydrogenation process; i.e., the removal of hydrogen atoms likely transforms the multilayer system from germanane to germanene, which is consistent with the drastic enhancement of conductivity and the insulator-to-metal transition.

In addition to the metallic state of multilayer germanene discussed above, theory also predicts a strong SOI for both germanene and germanane, ${ }^{32,58,59}$ which not only sets a good reference to further justify the aforementioned scenario but also makes multilayer germanene an attractive candidate for applications in spin-selective electronics. We measured the magnetoresistance (MR) with magnetic fields applied perpendicular to the $a b$-plane of germanane. As shown in Figure 4a, clear WAL is observed, manifesting as a characteristic sharp MR dip at $B=0 \mathrm{~T}$. According to the $2 \mathrm{D}$ localization theory, ${ }^{60-62}$ by assuming that the elastic and spin-orbit scattering times are much shorter than the inelastic scattering time, the magnetoconductivity $(\mathrm{MC})$ can be described by the following equation:

$$
\begin{aligned}
\Delta \sigma_{2 \mathrm{D}} & =\sigma_{2 \mathrm{D}}(B)-\sigma_{2 \mathrm{D}}(0) \\
& =-\frac{\alpha e^{2}}{2 \pi^{2} \hbar}\left[\operatorname{In} \frac{\hbar}{4 B \mathrm{e} l_{\Phi}{ }^{2}}-\psi\left(\frac{1}{2}+\frac{\hbar}{4 B \mathrm{e} l_{\Phi}{ }^{2}}\right)\right]
\end{aligned}
$$

where $\hbar$ is the reduced Planck constant, $e$ the charge of electron, $\psi(x)$ the digamma function, and $l_{\Phi}$ the phase coherence length. $\alpha$ is a fitting parameter equal to $+1,0$, and $-1 / 2$ for the orthogonal, unitary, and symplectic cases, respectively. ${ }^{60,63}$ The $\Delta \sigma_{2 \mathrm{D}}$ measured at $T=2.5 \mathrm{~K}$ is plotted in Figure $4 \mathrm{~b}$, where the solid curve represents the fitting with the above equation. We can see that the fitting matches very well with the experimental observation using parameters $\alpha=$ -0.53 and $l_{\Phi}=79 \mathrm{~nm}$. The clear WAL features provide strong evidence for strong SOI in annealed germanane.

It should be noted that the thermal property study of germanane in the previous report (Bianco et al. ACS Nano 2013, 7, 4414) also shows consistent observations with our study. In the diffuse reflectance absorption (DRA) spectroscopy, a significant red shift of the onset of optical absorption was observed after annealing, and the onset eventually decreased below that of bulk germanium. This result directly reveals the shrinking of semiconducting gap after annealing, which causes the enhancement of the electrical conductivity. Furthermore, in X-ray diffraction pattern, the $2 \theta$ value of the [002] reflection increased after annealing, directly implying a decrease of the interlayer distance along the $c$-axis.

To summarize, we measured the transport properties of annealed multilayer germanane, which has a layered structure similar to hydrogen-terminated graphene. Pristine germanane is insulating due to the finite gap in the band structure. We find that the conductivity can be gradually enhanced by thermal annealing in an Ar atmosphere, and a p-type field effect transistor is realized. After high temperature annealing, germanane becomes highly conductive and the temperature dependence of the resistance shows a metallic behavior. With a resistivity of $1.6 \times 10^{-7} \Omega \cdot \mathrm{m}$ at room temperature, a sharp $\mathrm{Ge}-\mathrm{Ge}$ vibrational mode at $300 \mathrm{~cm}^{-1}$ in the Raman spectra, and a uniform thickness decrease of $\sim 40 \%$, we suggest the product after annealing is most likely multilayer germanene, which has a structure similar to graphite. Clear weak antilocalization is observed at low temperatures with a phase coherence length of $\sim 80 \mathrm{~nm}$, providing strong evidence for strong SOI in annealed germanane. Realizing metallic transport with strong SOI paves the way for developing field effect transistors and spin- and optoelectronic devices based on annealed germanane. 


\section{ASSOCIATED CONTENT}

\section{S Supporting Information}

The Supporting Information is available free of charge on the ACS Publications website at DOI: 10.1021/acs.nanolett.8b04207.

Elemental analysis of germanane (using XPS, EDX, and SEM), annealing temperature dependence of conductivity from the same device, field effect tuning of the intermediate dehydrogenation state, transfer curve of annealed germanane in metallic state, and Hall measurement, carrier density, and mobility of germanane in metallic state (PDF)

\section{AUTHOR INFORMATION}

\section{Corresponding Author}

*E-mail: j.ye@rug.nl.

\section{ORCID}

Qihong Chen: 0000-0002-6039-0456

Petra Rudolf: 0000-0002-4418-1769

\section{Notes}

The authors declare no competing financial interest.

\section{ACKNOWLEDGMENTS}

We thank J. Harkema, A. Joshua, and J. Zoestbergen for technical support. Q. Chen thanks the scholarship from The Ubbo Emmius Fund. J. Ye and Q. Chen thank the Stichting voor Fundamenteel Onderzoek der Materie (FOM, FV157) and FlagERA iSpinText for financial support. J. Ye acknowledges funding from the European Research Council (consolidator Grant No. 648855, Ig-QPD).

\section{REFERENCES}

(1) Novoselov, K. S.; Geim, A. K.; Morozov, S. V.; Jiang, D.; Zhang, Y.; Dubonos, S. V.; Grigorieva, I. V.; Firsov, A. A. Electric Field Effect in Atomically Thin Carbon Films. Science 2004, 306 (5696), 666669.

(2) Novoselov, K. S.; Jiang, D.; Schedin, F.; Booth, T. J.; Khotkevich, V. V.; Morozov, S. V.; Geim, A. K. Two-Dimensional Atomic Crystals. Proc. Natl. Acad. Sci. U. S. A. 2005, 102 (30), 10451-10453.

(3) Liu, H.; Neal, A. T.; Zhu, Z.; Luo, Z.; Xu, X.; Tománek, D.; Ye, P. D. Phosphorene: An Unexplored 2D Semiconductor with a High Hole Mobility. ACS Nano 2014, 8 (4), 4033-4041.

(4) Mak, K. F.; Lee, C.; Hone, J.; Shan, J.; Heinz, T. F. Atomically Thin $\mathrm{MoS}_{2}$ : A New Direct-Gap Semiconductor. Phys. Rev. Lett. 2010, 105 (13), 136805.

(5) Ye, J. T.; Zhang, Y. J.; Akashi, R.; Bahramy, M. S.; Arita, R.; Iwasa, Y. Superconducting Dome in a Gate-Tuned Band Insulator. Science 2012, 338 (6111), 1193-1196.

(6) Wang, Q. H.; Kalantar-Zadeh, K.; Kis, A.; Coleman, J. N.; Strano, M. S. Electronics and Optoelectronics of Two-Dimensional Transition Metal Dichalcogenides. Nat. Nanotechnol. 2012, 7 (11), 699-712.

(7) Lu, J. M.; Zheliuk, O.; Leermakers, I.; Yuan, N. F. Q.; Zeitler, U.; Law, K. T.; Ye, J. T. Evidence for Two-Dimensional Ising Superconductivity in Gated MoS2. Science 2015, 350 (6266), $1353-1357$.

(8) Chen, Q.; Liang, L.; Ali El Yumin, A.; Lu, J.; Zheliuk, O.; Ye, J. High Quality Superconductor-Normal Metal Junction Made on the Surface of $\mathrm{MoS}_{2}$ Flakes. Phys. Status Solidi B 2017, 254, 1700181.

(9) Shi, W.; Ye, J.; Zhang, Y.; Suzuki, R.; Yoshida, M.; Miyazaki, J.; Inoue, N.; Saito, Y.; Iwasa, Y. Superconductivity Series in Transition Metal Dichalcogenides by Ionic Gating. Sci. Rep. 2015, 5, 12534.
(10) Chen, Q. H.; Lu, J. M.; Liang, L.; Zheliuk, O.; Ali, A.; Sheng, P.; Ye, J. T. Inducing and Manipulating Heteroelectronic States in a Single $\mathrm{MoS}_{2}$ Thin Flake. Phys. Rev. Lett. 2017, 119 (14), 147002.

(11) Chen, Q.; Lu, J.; Liang, L.; Zheliuk, O.; Ali El Yumin, A.; Ye, J. Continuous Low-Bias Switching of Superconductivity in a $\mathrm{MoS}_{2}$ Transistor. Adv. Mater. 2018, 30 (28), 1800399.

(12) Zhang, W. E.; Wang, F.-C.; Yang, C. H.; Yang, M. J. The Ultimate Scaling Limit of Semiconductor-Based Transistors. Superlattices Microstruct. 1997, 22 (3), 417-420.

(13) Kawaura, H.; Sakamoto, T.; Baba, T. Observation of Source-toDrain Direct Tunneling Current in $8 \mathrm{Nm}$ Gate Electrically Variable Shallow Junction Metal-Oxide-Semiconductor Field-Effect Transistors. Appl. Phys. Lett. 2000, 76 (25), 3810-3812.

(14) Lundstrom, M. Moore's Law Forever? Science 2003, 299 (5604), 210-211.

(15) Keyes, R. W. Physical Limits of Silicon Transistors and Circuits. Rep. Prog. Phys. 2005, 68 (12), 2701.

(16) Desai, S. B.; Madhvapathy, S. R.; Sachid, A. B.; Llinas, J. P.; Wang, Q.; Ahn, G. H.; Pitner, G.; Kim, M. J.; Bokor, J.; Hu, C.; Wong, H.-S. P.; Javey, A. $\mathrm{MoS}_{2}$ Transistors with 1-Nanometer Gate Lengths. Science 2016, 354 (6308), 99-102.

(17) Aufray, B.; Kara, A.; Vizzini, S.; Oughaddou, H.; Léandri, C.; Ealet, B.; Le Lay, G. Graphene-like Silicon Nanoribbons on $\operatorname{Ag}(110)$ : A Possible Formation of Silicene. Appl. Phys. Lett. 2010, 96 (18), 183102.

(18) Lalmi, B.; Oughaddou, H.; Enriquez, H.; Kara, A.; Vizzini, S.; Ealet, B.; Aufray, B. Epitaxial Growth of a Silicene Sheet. Appl. Phys. Lett. 2010, 97 (22), 223109.

(19) Tao, L.; Cinquanta, E.; Chiappe, D.; Grazianetti, C.; Fanciulli, M.; Dubey, M.; Molle, A.; Akinwande, D. Silicene Field-Effect Transistors Operating at Room Temperature. Nat. Nanotechnol. 2015, 10 (3), 227-231.

(20) Chen, L.; Liu, C.-C.; Feng, B.; He, X.; Cheng, P.; Ding, Z.; Meng, S.; Yao, Y.; Wu, K. Evidence for Dirac Fermions in a Honeycomb Lattice Based on Silicon. Phys. Rev. Lett. 2012, 109 (5), 056804.

(21) Vogt, P.; De Padova, P.; Quaresima, C.; Avila, J.; Frantzeskakis, E.; Asensio, M. C.; Resta, A.; Ealet, B.; Le Lay, G. Silicene: Compelling Experimental Evidence for Graphenelike Two-Dimensional Silicon. Phys. Rev. Lett. 2012, 108 (15), 155501.

(22) Guzmán-Verri, G. G.; Lew Yan Voon, L. C. Electronic Structure of Silicon-Based Nanostructures. Phys. Rev. B: Condens. Matter Mater. Phys. 2007, 76 (7), 075131.

(23) Lebègue, S.; Eriksson, O. Electronic Structure of TwoDimensional Crystals from ab Initio Theory. Phys. Rev. B: Condens. Matter Mater. Phys. 2009, 79 (11), 115409.

(24) Ni, Z.; Liu, Q.; Tang, K.; Zheng, J.; Zhou, J.; Qin, R.; Gao, Z.; Yu, D.; Lu, J. Tunable Bandgap in Silicene and Germanene. Nano Lett. 2012, 12 (1), 113-118.

(25) Dávila, M. E.; Xian, L.; Cahangirov, S.; Rubio, A.; Le Lay, G. Germanene: A Novel Two-Dimensional Germanium Allotrope Akin to Graphene and Silicene. New J. Phys. 2014, 16 (9), 095002.

(26) Dávila, M. E.; Le Lay, G. Few Layer Epitaxial Germanene: A Novel Two-Dimensional Dirac. Sci. Rep. 2016, 6, 20714.

(27) Zhang, L.; Bampoulis, P.; Rudenko, A. N.; Yao, Q.; van Houselt, A.; Poelsema, B.; Katsnelson, M. I.; Zandvliet, H. J. W. Structural and Electronic Properties of Germanene on $\mathrm{MoS}_{2}$. Phys. Rev. Lett. 2016, 116 (25), 256804.

(28) Acun, A.; Zhang, L.; Bampoulis, P.; Farmanbar, M.; vanHouselt, A.; Rudenko, A. N.; Lingenfelder, M.; Brocks, G.; Poelsema, B.; Katsnelson, M. I.; Zandvliet, H. J. W. Germanene: The Germanium Analogue of Graphene. J. Phys.: Condens. Matter 2015, 27 (44), 443002.

(29) Elias, D. C.; Nair, R. R.; Mohiuddin, T. M. G.; Morozov, S. V.; Blake, P.; Halsall, M. P.; Ferrari, A. C.; Boukhvalov, D. W.; Katsnelson, M. I.; Geim, A. K.; Novoselov, K. S. Control of Graphene's Properties by Reversible Hydrogenation: Evidence for Graphane. Science 2009, 323 (5914), 610-613. 
(30) Sofo, J. O.; Chaudhari, A. S.; Barber, G. D. Graphane: A TwoDimensional Hydrocarbon. Phys. Rev. B: Condens. Matter Mater. Phys. 2007, 75 (15), 153401.

(31) Bianco, E.; Butler, S.; Jiang, S.; Restrepo, O. D.; Windl, W.; Goldberger, J. E. Stability and Exfoliation of Germanane: A Germanium Graphane Analogue. ACS Nano 2013, 7 (5), 4414-4421.

(32) Amamou, W.; Odenthal, P. M.; Bushong, E. J.; O’Hara, D. J.; Luo, Y. K.; van Baren, J.; Pinchuk, I.; Wu, Y.; Ahmed, A. S.; Katoch, J.; Bockrath, M. W.; Tom, H. W. K.; Goldberger, J. E.; Kawakami, R. K. Large Area Epitaxial Germanane for Electronic Devices. 2D Mater. 2015, 2 (3), 035012.

(33) Luo, X.; Zurek, E. Crystal Structures and Electronic Properties of Single-Layer, Few-Layer, and Multilayer GeH. J. Phys. Chem. C 2016, 120 (1), 793-800.

(34) Voon, L. C. L. Y.; Sandberg, E.; Aga, R. S.; Farajian, A. A. Hydrogen Compounds of Group-IV Nanosheets. Appl. Phys. Lett. 2010, 97 (16), 163114.

(35) Houssa, M.; Scalise, E.; Sankaran, K.; Pourtois, G.; Afanas'ev, V. V.; Stesmans, A. Electronic Properties of Hydrogenated Silicene and Germanene. Appl. Phys. Lett. 2011, 98 (22), 223107.

(36) Lebègue, S.; Klintenberg, M.; Eriksson, O.; Katsnelson, M. I. Accurate Electronic Band Gap of Pure and Functionalized Graphane from GW Calculations. Phys. Rev. B: Condens. Matter Mater. Phys. 2009, 79 (24), 245117.

(37) Zhao, Y.; Yin, D.; Yoon, Y. Intrinsic Performance of Germanane Schottky Barrier Field-Effect Transistors. IEEE Trans. Electron Devices 2018, 65, 4188-4195.

(38) Yao, Q.; Zhang, L.; Kabanov, N. S.; Rudenko, A. N.; Arjmand, T.; Rahimpour Soleimani, H.; Klavsyuk, A. L.; Zandvliet, H. J. W. Bandgap Opening in Hydrogenated Germanene. Appl. Phys. Lett. 2018, 112 (17), 171607.

(39) Liu, C.-C.; Feng, W.; Yao, Y. Quantum Spin Hall Effect in Silicene and Two-Dimensional Germanium. Phys. Rev. Lett. 2011, 107 (7), 076802.

(40) Sahoo, N. G.; Esteves, R. J.; Punetha, V. D.; Pestov, D.; Arachchige, I. U.; McLeskey, J. T., Jr. Schottky Diodes from 2D Germanane. Appl. Phys. Lett. 2016, 109 (2), 023507.

(41) Young, J. R.; Chitara, B.; Cultrara, N. D.; Arguilla, M. Q.; Jiang, S.; Fan, F.; Johnston-Halperin, E.; Goldberger, J. E. Water Activated Doping and Transport in Multilayered Germanane Crystals. J. Phys.: Condens. Matter 2016, 28 (3), 034001.

(42) Madhushankar, B. N.; Kaverzin, A.; Giousis, T.; Potsi, G.; Gournis, D.; Rudolf, P.; Blake, G. R.; van der Wal, C. H.; van Wees, B. J. Electronic Properties of Germanane Field-Effect Transistors. 2D Mater. 2017, 4 (2), 021009.

(43) Cultrara, N. D.; Wang, Y.; Arguilla, M. Q.; Scudder, M. R.; Jiang, S.; Windl, W.; Bobev, S.; Goldberger, J. E. Synthesis of 1T, 2H, and 6R Germanane Polytypes. Chem. Mater. 2018, 30 (4), 13351343.

(44) Chaudhuri, S.; Shen, L.; Healy, N.; Peacock, A. C.; Badding, J. V. Hydrogenated Amorphous Germanium Optical Fiber. Novel Optical Materials and Applications; Optical Society of America, 2015; NT2C.4.

(45) Wihl, M.; Cardona, M.; Tauc, J. Raman Scattering in Amorphous Ge and III-V Compounds. J. Non-Cryst. Solids 1972, $8,172-178$.

(46) Bermejo, D.; Cardona, M. Raman Scattering in Pure and Hydrogenated Amorphous Germanium and Silicon. J. Non-Cryst. Solids 1979, 32 (1), 405-419.

(47) Veldhuizen, L. W.; van der Werf, C. H. M.; Kuang, Y.; Bakker, N. J.; Yun, S. J.; Schropp, R. E. I. Optimization of Hydrogenated Amorphous Silicon Germanium Thin Films and Solar Cells Deposited by Hot Wire Chemical Vapor Deposition. Thin Solid Films 2015, 595, 226-230.

(48) Hung, C. S.; Gliessman, J. R. Resistivity and Hall Effect of Germanium at Low Temperatures. Phys. Rev. 1954, 96 (5), 12261236.

(49) Clark, A. H. Electrical and Optical Properties of Amorphous Germanium. Phys. Rev. 1967, 154 (3), 750-757.
(50) Trumbore, F. A.; Tartaglia, A. A. Resistivities and Hole Mobilities in Very Heavily Doped Germanium. J. Appl. Phys. 1958, 29 (10), 1511-1511.

(51) Tyler, W. W.; Wilson, A. C. Thermal Conductivity, Electrical Resistivity, and Thermoelectric Power of Graphite. Phys. Rev. 1953, 89 (4), 870-875.

(52) Chen, J.-H.; Jang, C.; Xiao, S.; Ishigami, M.; Fuhrer, M. S. Intrinsic and Extrinsic Performance Limits of Graphene Devices on $\mathrm{SiO}_{2}$. Nat. Nanotechnol. 2008, 3 (4), 206-209.

(53) Sharma, S. K.; Jain, S. C.; Aggarwal, S. S.; Bhide, V. G. Structure and Electrical Resistivity of Thin Amorphous Germanium Films. J. Non-Cryst. Solids 1972, 7 (3), 285-294.

(54) Pollak, M.; Knotek, M. L.; Kurtzman, H.; Glick, H. Dc Conductivity of Amorphous Germanium and the Structure of the Pseudogap. Phys. Rev. Lett. 1973, 30 (18), 856-859.

(55) Cápek, V.; Koc, S.; Zemek, J. DC Conductivity of Amorphous Germanium. J. Non-Cryst. Solids 1975, 18 (1), 95-106.

(56) Thieme, W.; Diletti, H.; Coufal, H.; Gierisch, W.; Lüscher, E. The Electrical Resistivity of Highly Doped P-Type Germanium from $40 \mathrm{mK}$ to $300 \mathrm{~K}$. J. Less-Common Met. 1978, 62, 405-406.

(57) Cahangirov, S.; Topsakal, M.; Aktürk, E.; Şahin, H.; Ciraci, S. Two- and One-Dimensional Honeycomb Structures of Silicon and Germanium. Phys. Rev. Lett. 2009, 102 (23), 236804.

(58) Seixas, L.; Padilha, J. E.; Fazzio, A. Quantum Spin Hall Effect on Germanene Nanorod Embedded in Completely Hydrogenated Germanene. Phys. Rev. B: Condens. Matter Mater. Phys. 2014, 89 (19), 195403.

(59) Si, C.; Liu, J.; Xu, Y.; Wu, J.; Gu, B.-L.; Duan, W. Functionalized Germanene as a Prototype of Large-Gap TwoDimensional Topological Insulators. Phys. Rev. B: Condens. Matter Mater. Phys. 2014, 89 (11), 115429.

(60) Hikami, S.; Larkin, A. I.; Nagaoka, Y. Spin-Orbit Interaction and Magnetoresistance in the Two Dimensional Random System. Prog. Theor. Phys. 1980, 63 (2), 707-710.

(61) McCann, E.; Kechedzhi, K.; Fal'ko, V. I.; Suzuura, H.; Ando, T.; Altshuler, B. L. Weak-Localization Magnetoresistance and Valley Symmetry in Graphene. Phys. Rev. Lett. 2006, 97 (14), 146805.

(62) He, H.-T.; Wang, G.; Zhang, T.; Sou, I.-K.; Wong, G. K. L.; Wang, J.-N.; Lu, H.-Z.; Shen, S.-Q.; Zhang, F.-C. Impurity Effect on Weak Antilocalization in the Topological Insulator $\mathrm{Bi}_{2} \mathrm{Te}_{3}$. Phys. Rev. Lett. 2011,106 (16), 166805.

(63) Dyson, F. J. Statistical Theory of the Energy Levels of Complex Systems. I. J. Math. Phys. 1962, 3 (1), 140-156. 